\title{
Scattering and absorption properties of polydisperse wavelength-sized particles covered with much smaller grains
}

\author{
Janna M. Dlugach ${ }^{\mathrm{a}}$, Michael I. Mishchenko ${ }^{\mathrm{b}, *}$, Daniel W. Mackowski ${ }^{\mathrm{c}}$ \\ a Main Astronomical Observatory of the National Academy of Sciences of Ukraine, 27 Zabolotny Street, 03680 Kyiv, Ukraine \\ b NASA Goddard Institute for Space Studies, 2880 Broadway, New York, NY 10025, USA \\ ' Department of Mechanical Engineering, Auburn University, AL 36849, USA
}

\section{A R T I C L E I N F O}

Available online 17 March 2012

Keywords:

Maxwell equations

Electromagnetic scattering

Polarization

Scattering and absorption characteristics

Small-scale surface roughness

Wavelength-sized particles

\begin{abstract}
A B S T R A C T
Using the results of direct, numerically exact computer solutions of the Maxwell equations, we analyze scattering and absorption characteristics of polydisperse compound particles in the form of wavelength-sized spheres covered with a large number of much smaller spherical grains. The results pertain to the complex refractive indices $1.55+\mathrm{i} 0.0003,1.55+\mathrm{i} 0.3$, and $3+\mathrm{i} 0.1$. We show that the optical effects of "dusting" wavelength-sized hosts by microscopic grains can vary depending on the number and size of the grains as well as on the complex refractive index. Our computations also demonstrate the high efficiency of the new superposition $T$-matrix code developed for use on distributed memory computer clusters.
\end{abstract}

Published by Elsevier Ltd.

\section{Introduction}

The growing efficiency of computers and numerically exact codes based on direct solutions of the Maxwell equations allows one to study scattering and absorption properties of small particles with increasingly complex morphologies [1-11]. In recent papers [12,13], we introduced a new particle morphology in the form of a wavelength-sized spherical host covered with a large number of much smaller spherical grains. This morphology can be used to study the potential optical effects of microscopic surface irregularities of wavelength-sized particles (cf. [14-24]) as well as to model the actual "dusting" of wavelength-sized particles by microscopic grains [13].

The analysis in Ref. [12] was limited to one refractive index typical of mineral aerosols $(m=1.55+\mathrm{i} 0.0003$, where $\mathrm{i}=(-1)^{1 / 2}$ ) and to fewer than 50 small grains covering a large host. This paper is a natural extension of Ref. [12] in two respects. First, we analyze the effect of

\footnotetext{
* Corresponding author. Tel.: +1 212678 5590; fax: +1212678 5622 .

E-mail addresses: michael.i.mishchenko@nasa.gov, crmim2@gmail.com (M.I. Mishchenko).
}

a five-fold increase in the number of small grains, whereupon the grains cover the surface of the large hosts almost entirely. Second, we analyze the consequences of increasing absorption $(m=1.55+\mathrm{i} 0.3)$ as well as increasing both the real and the imaginary parts of the refractive index $(m=3+\mathrm{i} 0.1)$. A related objective is to further assess the efficiency and versatility of the new public-domain Fortran-90 code based on the superposition $T$-matrix method (STMM) [25] and developed for use on distributed memory computer clusters [26,27].

\section{Numerically exact computer modeling}

Following Ref. [12], we consider a scattering target obtained by placing a number $N_{\mathrm{g}}$ of small non-overlapping spherical grains of radius $r$ randomly on the surface of a relatively large spherical host of radius $R$ using a random number generator (see the inset in Fig. 1(a)). The refractive indices of the host and the grains are the same. It is assumed that the target is illuminated by a parallel quasi-monochromatic beam of light and that the observation point is located in the far-field zone. It is also assumed that all scattering and absorption characteristics are averaged over the uniform orientation distribution of 
the resulting host-grains configuration with respect to the laboratory coordinate system. The transformation of the Stokes parameters upon far-field electromagnetic scattering is then written in terms of the normalized Stokes scattering matrix [28]:

$$
\left[\begin{array}{l}
I^{\text {sca }} \\
Q^{\text {sca }} \\
U^{\text {sca }} \\
V^{\text {sca }}
\end{array}\right] \propto\left[\begin{array}{cccc}
a_{1}(\theta) & b_{1}(\theta) & 0 & 0 \\
b_{1}(\theta) & a_{2}(\theta) & 0 & 0 \\
0 & 0 & a_{3}(\theta) & b_{2}(\theta) \\
0 & 0 & -b_{2}(\theta) & a_{4}(\theta)
\end{array}\right]\left[\begin{array}{c}
I^{\text {inc }} \\
Q^{\text {inc }} \\
U^{\text {inc }} \\
V^{\text {inc }}
\end{array}\right],
$$

where $\theta \in\left[0^{\circ}, 180^{\circ}\right]$ is the angle between the incidence and scattering directions, while both sets of the Stokes parameters are defined with respect to the scattering plane. Strictly speaking, the off-block diagonal elements of the scattering matrix vanish only if the compound particle has a plane of symmetry. We have found, however, that in the cases considered, the elements denoted by zeros are negligibly small (in the absolute-value sense) in comparison with the other elements at the same scattering angles. The $(1,1)$ element $a_{1}(\theta)$, called the phase function, is normalized according to the integral condition

$\frac{1}{2} \int_{0}^{\pi} a_{1}(\theta) \sin \theta d \theta=1$.

This model of compound aerosols allows us to compute all scattering and absorption characteristics using the highly efficient and numerically exact STMM. Specifically, we used the recently developed parallelized computer program posted on-line [27] to perform extensive computations for different values of $N_{\mathrm{g}}$ and $\mathrm{m}$. Half of our results pertain to the refractive index $1.55+\mathrm{i} 0.0003$ typical of mineral aerosols in the visible part of the spectrum. In order to analyze the effect of increasing $N_{\mathrm{g}}$, we consider the values $N_{\mathrm{g}}=0,49,149$, and 249 . To study the effect of increasing absorption, computations were also performed for $m=1.55+\mathrm{i} 0.3$. Finally, we consider the case of hematite particles with $m=3+\mathrm{i} 0.1$ (cf. Refs. [21,23]). In the majority of simulations, the ratio of the host radius to that of the small grains was kept constant at $R / r=10$. However, to analyze the effect of increasing grain size relative to that of the host, we also considered compound particles with $m=1.55+\mathrm{i} 0.0003$ and $R / r=5$. The wavelength was kept constant at $0.62832 \mu \mathrm{m}$.

To eliminate resonances typical of monodisperse particles [16,28], all scattering and absorption characteristics were averaged over the standard power-law distribution of host radii [28]:

$n(R)=\left\{\begin{array}{cc}\text { constant } \times R^{-3}, & R_{\min } \leq R \leq R_{\max } \\ 0 & \text { otherwise }\end{array}\right.$

with an effective radius of $R_{\text {eff }}=1 \mu \mathrm{m}$ and an effective variance of $v_{\text {eff }}=0.05$. Of course, averaging over sizes also renders a better representation of polydisperse particle ensembles encountered in various applications. The averaging was based on the Gauss-Legendre quadrature formula with 100 division points applied to the corresponding range of host radii $0.661 \mu \mathrm{m} \leq R \leq 1.439 \mu \mathrm{m}$. The corresponding effective radius of the small grains (equal to $1 / 5$ or $1 / 10$ times $R_{\text {eff }}$ ) will be denoted by $r_{\text {eff. }}$

\section{Numerical results and discussion}

Table 1 lists ensemble-averaged integral radiometric characteristics, viz., the extinction, $C_{\text {ext }}$, scattering, $C_{\mathrm{sca}}$, and absorption, $C_{\mathrm{abs}}$, cross sections as well as the single-scattering albedo $\varpi$ and the asymmetry parameter $g$. The data presented in the first four rows of the table show how these characteristics change with $N_{\mathrm{g}}$ in the case of $m=1.55+\mathrm{i} 0.0003$. It is seen that all three cross sections increase, especially $C_{\text {ext }}$ and $C_{\text {sca }}$, while the single-scattering albedo and the asymmetry parameter hardly change. The significant increase of the extinction and scattering cross sections can be attributed primarily to the growing geometrical cross sections of the compound particles. Increasing absorption in both the hosts and the small grains $\left(m=1.55+\mathrm{i} 0.3, N_{\mathrm{s}}=0\right.$ and 49$)$ results in a non-negligible increase of all three cross sections and a decrease of the single-scattering albedo with increasing $N_{\mathrm{g}}$ (compare the sixth and seventh rows). Comparison of the first, second, and fifth rows reveals a much stronger effect of dusting the large hosts with $R / r=5$ grains. All three cross sections increase quite substantially, whereas the asymmetry parameter is significantly reduced. Finally, the last two rows of Table 1 reveal rather modest changes in the integral radiometric characteristics upon covering $R_{\text {eff }}=1$ hematite hosts with $N_{\mathrm{g}}=49$ small $(r / R=0.1)$ hematite grains.

Fig. 1 parallels Table 1 and depicts non-zero elements of the Stokes scattering matrix for both "clean" and "contaminated" hosts. Fig. 1(a) shows that the effect of increasing $N_{\mathrm{g}}$ on the phase function is three-fold: (i) the forward-scattering diffraction peak becomes somewhat stronger; (ii) there is a modest increase at side-scattering angles; and (iii) the backscattering peak is suppressed substantially, the differences between the $N_{\mathrm{g}}=0$ and $N_{\mathrm{g}}=249$ phase functions exceeding a factor of 4 . By the time $N_{\mathrm{g}}$ reaches the value 249, the small grains form a quasi-uniform coating around the hosts, thereby making the compound particles quasi-spherical with $R_{\mathrm{eff}} \approx 1.2 \mu \mathrm{m}$. However, the resulting increase in the particles' geometrical cross sections can explain only trend (i), as the gray curve in the upper left panel of Fig. 1(a) vividly demonstrates. Trends (ii) and (iii) are, in fact, reminiscent of the typical effects of increasing asphericity of such morphologically simple particles as spheroids [28-30]. The phase-function results computed for the case of $R / r=5$ and $N_{\mathrm{g}}=49$ are even more indicative of these effects (see the dark blue curve in the upper left panel of Fig. 1(a)), which is not surprising given the substantially larger size of the grains leading to visibly more pronounced asphericity of the resulting compounded particles. The linear-polarization curves in Fig. 1(a) reveal another typical effect of increasing asphericity resulting in progressively neutral polarization [31]. The $-b_{1}(\theta) / a_{1}(\theta)$ curve plotted for $R / r=5$ and $N_{g}=49$ even appears to show the gradual development of a positive-polarization maximum at side- and near-backscattering angles typical of spheroids and natural mineral aerosols [31-33].

The upper right-hand panel of Fig. 1(a) depicts the computed ratio $a_{2}(\theta) / a_{1}(\theta)$, which is identically equal to $100 \%$ for "clean" spherical hosts but deviates from $100 \%$ for contaminated particles, thereby signifying the nonsphericity of the latter and causing non-zero linear 
a

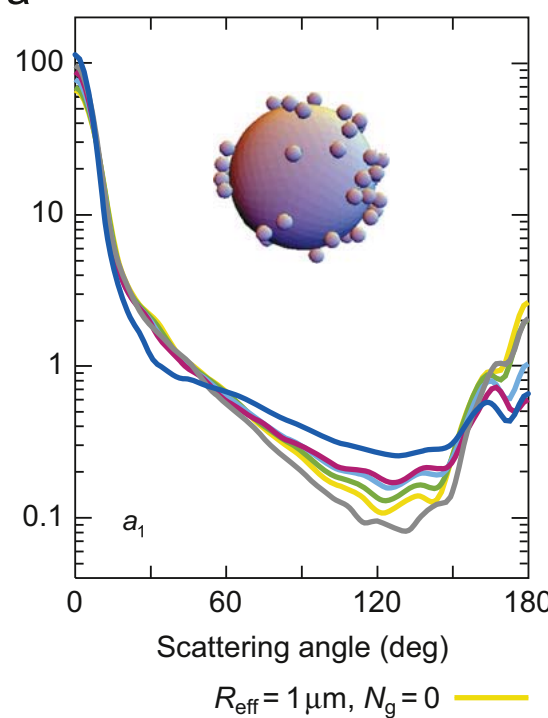

$R_{\text {eff }}=1 \mu \mathrm{m}, N_{\mathrm{g}}=49, r_{\text {eff }}=0.1 \mu \mathrm{m}$

$R_{\text {eff }}=1 \mu \mathrm{m}, N_{\mathrm{g}}=149, r_{\text {eff }}=0.1 \mu \mathrm{m}$

$R_{\text {eff }}=1 \mu \mathrm{m}, N_{\mathrm{g}}=249, r_{\mathrm{eff}}=0.1 \mu \mathrm{m}$

$R_{\text {eff }}=1.2 \mu \mathrm{m}, N_{\mathrm{g}}=0$

$R_{\text {eff }}=1 \mu \mathrm{m}, N_{\mathrm{g}}=49, r_{\text {eff }}=0.2 \mu \mathrm{m}$

b

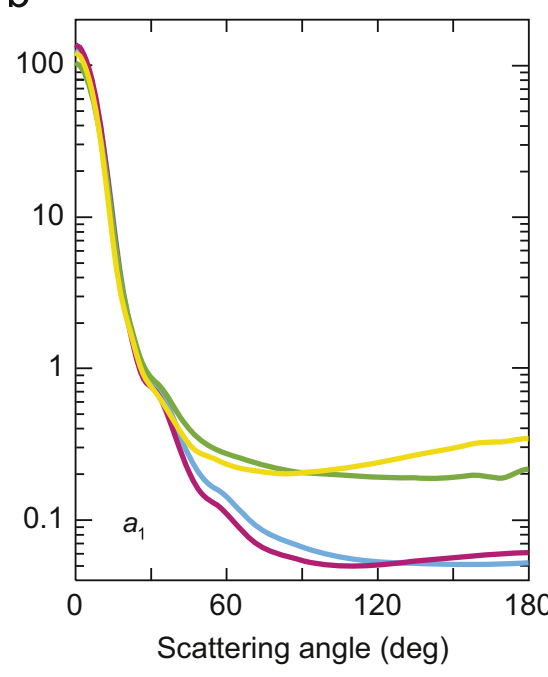

$m=1.55+\mathrm{i} 0.3, N_{\mathrm{g}}=0$

$m=1.55+\mathrm{i} 0.3, N_{\mathrm{g}}=49$

$m=3+\mathrm{i} 0.1, N_{\mathrm{g}}=0$

$m=3+\mathrm{i} 0.1, N_{\mathrm{g}}=49$
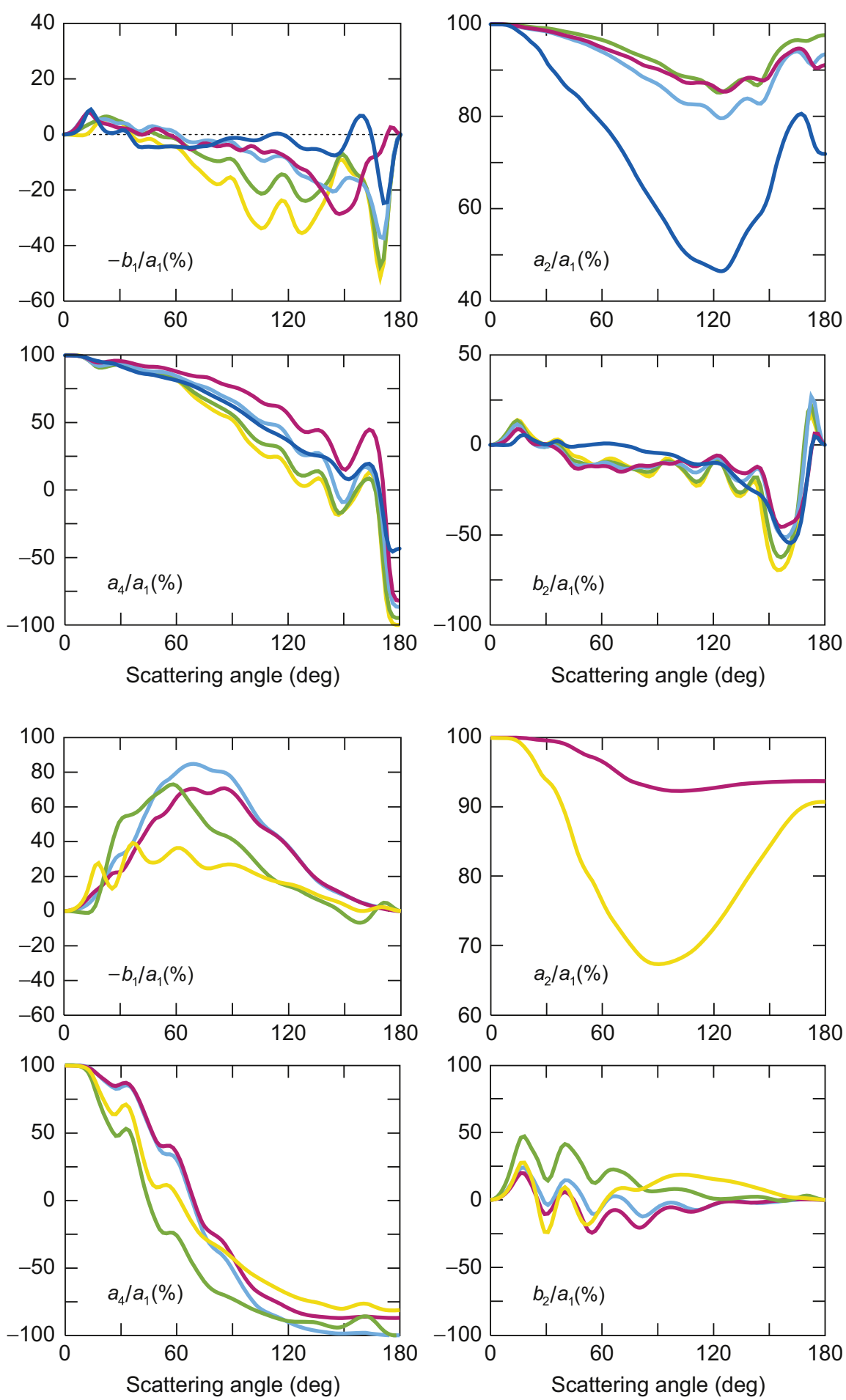

Fig. 1. (a) Elements of the normalized Stokes scattering matrix for "clean" polydisperse hosts and polydisperse hosts "dusted" with different numbers of small grains. The refractive index is $1.55+\mathrm{i} 0.0003$. The inset illustrates the morphology of the compound particles for $N_{\mathrm{g}}=49$. (b) As in (a), but for refractive indices $1.55+\mathrm{i} 0.3$ and $3+\mathrm{i} 0.1$. The effective radii of the hosts and the small grains are fixed at $1 \mu \mathrm{m}$ and $0.1 \mu \mathrm{m}$, respectively.

depolarization ratios [34]. The deviation of the ratio $a_{2}(\theta) /$ $a_{1}(\theta)$ from $100 \%$ is especially large in the case of $R / r=5$ and $N_{\mathrm{g}}=49$, which can be explained qualitatively as being the consequence of the greater overall asphericity of the resulting compound particles and stronger "electromagnetic interactions" between the grains and the host.
The most obvious manifestation of the nonsphericity of the compound particles in the $a_{4}(\theta) / a_{1}(\theta)$ curves is their deviation from $-100 \%$ at $\theta=180^{\circ}$, causing non-zero circular depolarization ratios [34]. Again, this deviation is especially large for compound particles with $R / r=5$ and $N_{\mathrm{g}}=49$. On the other hand, the numerical data depicted in 
Table 1

Ensemble-averaged extinction, scattering, and absorption cross sections per compound aerosol particle, single-scattering albedo $\varpi$, and asymmetry parameter $g$ as functions of the number $N_{g}$ of small grains covering the surface of a large host.

\begin{tabular}{|c|c|c|c|c|c|c|c|}
\hline$m$ & $r_{\text {eff }} / R_{\text {eff }}$ & $N_{\mathrm{g}}$ & $C_{\mathrm{ext}}\left(\mu \mathrm{m}^{2}\right)$ & $C_{\mathrm{sca}}\left(\mu \mathrm{m}^{2}\right)$ & $C_{\mathrm{abs}}\left(\mu \mathrm{m}^{2}\right)$ & $\varpi$ & $g$ \\
\hline $1.55+\mathrm{i} 0.0003$ & - & 0 & 6.314 & 6.265 & 0.049 & 0.9923 & 0.668 \\
\hline $1.55+\mathrm{i} 0.0003$ & 0.1 & 49 & 6.607 & 6.558 & 0.049 & 0.9926 & 0.661 \\
\hline $1.55+\mathrm{i} 0.0003$ & 0.1 & 149 & 7.358 & 7.304 & 0.054 & 0.9927 & 0.661 \\
\hline $1.55+\mathrm{i} 0.0003$ & 0.1 & 249 & 8.214 & 8.156 & 0.058 & 0.9931 & 0.669 \\
\hline $1.55+\mathrm{i} 0.0003$ & 0.2 & 49 & 10.670 & 10.606 & 0.064 & 0.9940 & 0.609 \\
\hline $1.55+\mathrm{i} 0.3$ & - & 0 & 6.464 & 3.183 & 3.281 & 0.492 & 0.912 \\
\hline $1.55+\mathrm{i} 0.3$ & 0.1 & 49 & 6.845 & 3.265 & 3.580 & 0.477 & 0.920 \\
\hline $3+\mathrm{i} 0.1$ & - & 0 & 7.355 & 4.312 & 3.043 & 0.586 & 0.776 \\
\hline $3+\mathrm{i} 0.1$ & 0.1 & 49 & 7.448 & 4.408 & 3.040 & 0.592 & 0.736 \\
\hline
\end{tabular}

the bottom right-hand panel of Fig. 1(a) demonstrate rather insignificant changes of the ratio $b_{2}(\theta) / a_{1}(\theta)$ with increasing $N_{\mathrm{g}}$ and/or increasing grain size.

In order to analyze the effect of increasing absorption, in Fig. 1(b) we present the numerical data obtained for $R_{\text {eff }}=1 \mu \mathrm{m}, R / r=10, N_{\mathrm{g}}=0$ and 49 , and $m=1.55+\mathrm{i} 0.3$. Comparison with the analogous cases in Fig. 1(a) reveals a similar magnitude of changes caused by the presence of $N_{\mathrm{g}}=49$ grains on the surfaces of much larger hosts.

Finally, the combined effects of increasing both the real and the imaginary parts of the refractive index are revealed by the results of computations for "clean" and "contaminated" hematite particles with $R_{\mathrm{eff}}=1 \mu \mathrm{m}$, $R / r=10, N_{\mathrm{g}}=0$ and 49 , and $m=3+\mathrm{i} 0.1$ (Fig. 2(b)). In contrast to the integral radiometric characteristics discussed above, dusting each large host with 49 small grains causes a noticeable change in all elements of the scattering matrix. The deviation of the ratio $a_{2}(\theta) / a_{1}(\theta)$ from $100 \%$ at side-scattering angles is especially pronounced.

\section{Concluding remarks}

This paper in combination with Refs. [12,13] demonstrates that large host particles covered with a large number of microscopic grains represent an interesting morphological class of wavelength-sized scatterers which can be used to model real "dusting" of larger aerosols by much smaller ones or, possibly, the optical effects of microscopic surface irregularities. In the case of a modest number of grains and a small real part of the refractive index, the optical consequences of such "contamination" are relatively weak. They become more pronounced with increasing real part of the refractive index, with increasing grain size relative to the host's size, and/or in cases when the smaller grains cover a large fraction of the host's surface. In some respects the optical effects of increasing the number of grains or the $r / R$ ratio in the case of the refractive index $1.55+\mathrm{i} 0.0003$ appear to be qualitatively similar to those of increasing the aspect ratio of spheroids, although a quantitative analysis of this similarity is problematic given the obvious geometrical incompatibility of these two types of particle morphology. By far the greatest are the effects of surface contamination of weakly absorbing mineral hosts by strongly absorbing soot grains [13].

A natural byproduct of our study was a further assessment of the utility of the new Fortran-90 STMM code $[26,27]$ when run on distributed memory computer clusters. Based on numerous timing tests, we conclude that the requisite computer time is inversely proportional to the number of nodes and to the number of cores in each node. Overall, our extensive experience with the old and new versions of the STMM code demonstrates that the latter is both highly accurate and very efficient.

\section{Acknowledgments}

This research was partly funded by the NASA Remote Sensing Theory Program managed by Lucia Tsaoussi and by the NASA Radiation Sciences Program managed by Hal Maring. We acknowledge support from the National Academy of Sciences of Ukraine under the Main Astronomical Observatory GRAPE/GPU/GRID Computing Cluster Project. JMD is also grateful to the organizers of the ELS-XIII conference for providing financial travel support.

\section{References}

[1] Hovenier JW, editor. Light scattering by non-spherical particles. J Quant Spectrosc Radiat Transfer 1996; 55:535-694.

[2] Lumme K, editor. Light scattering by non-spherical particles. J Quant Spectrosc Radiat Transfer 1998;60:301-500.

[3] Mishchenko MI, Hovenier JW, Travis LD, editors. Light scattering by nonspherical particles '98. J Quant Spectrosc Radiat Transfer 1999;63:127-738.

[4] Videen G, Fu Q, Chýlek P, editors. Light scattering by non-spherical particles. J Quant Spectrosc Radiat Transfer 2001;70:373-831.

[5] Kolokolova L, Gustafson BÅS, Mishchenko MI, Videen G, editors. Electromagnetic and light scattering by nonspherical particles 2002. J Quant Spectrosc Radiat Transfer 2003;79-80:491-1198.

[6] Wriedt T, editor. VII electromagnetic and light scattering by nonspherical particles: theory, measurement and applications. J Quant Spectrosc Radiat Transfer 2004;89:1-460.

[7] Moreno F, Muñoz O, López-Moreno JJ, Molina A, editors. VIII conference on electromagnetic and light scattering by nonspherical particles. J Quant Spectrosc Radiat Transfer 2006;100:1-495.

[8] Voshchinnikov NV, Videen G, editors. IX conference on electromagnetic and light scattering by non-spherical particles. J Quant Spectrosc Radiat Transfer 2007;106:1-621.

[9] Mishchenko MI, Videen G, Mengüç MP, editors. X conference on electromagnetic and light scattering by non-spherical particles. J Quant Spectrosc Radiat Transfer 2008;109:1335-548.

[10] Hough J, editor. XI conference on electromagnetic and light scattering by non-spherical particles. J Quant Spectrosc Radiat Transfer 2009;110:1207-779.

[11] Muinonen K, Videen G, Nousiainen T, Zubko E, Penttilä A, editors Electromagnetic and light scattering by nonspherical particles XII. J Quant Spectrosc Radiat Transfer 2011;112:1633-1929.

[12] Mishchenko MI, Dlugach JM, Mackowski DW. Light scattering by wavelength-sized particles "dusted" with subwavelength-sized grains. Opt Lett 2011;36:337-9. 
[13] Mishchenko MI, Dlugach JM. Adhesion of mineral and soot aerosols can strongly affect their scattering and absorption properties. Opt Lett 2012;37:704-6.

[14] Muinonen $K$, Nousiainen T, Lindqvist $H$, et al. Light scattering by Gaussian particles with internal inclusions and roughened surfaces using ray optics. J Quant Spectrosc Radiat Transfer 2009;110: 1628-39.

[15] Nousiainen T, Muñoz O, Lindqvist $H$, et al. Scattering of light by mineral-dust particles much larger than the wavelength. In: Muinonen K., Penttila A., Lindqvist H., et al., editors. Conference proceedings of electromagnetic and light scattering XII. University of Helsinki, Finland; 2010. p. 206-9.

[16] Mishchenko MI, Lacis AA. Morphology-dependent resonances of nearly spherical particles in random orientation. Appl Opt 2003;42: 5551-6.

[17] Li C, Kattawar GW, Yang P. Effects of surface roughness on light scattering by small particles. J Quant Spectrosc Radiat Transfer 2004;89:123-31.

[18] Rother T, Schmidth K, Wauer J, et al. Light scattering on Chebyshev particles of higher order. Appl Opt 2006;45:6030-7.

[19] Nousiainen T, Muinonen K. Surface-roughness effects on singlescattering properties of wavelength-scale particles. J Quant Spectrosc Radiat Transfer 2007;106:389-97.

[20] Zubko E, Muinonen K, Shkuratov Y, et al. Scattering of light by roughened Gaussian random particles. J Quant Spectrosc Radiat Transfer 2007;106:604-15.

[21] Kahnert M, Nousiainen T, Mauno P. On the impact of non-sphericity and small-scale surface roughness on the optical properties of hematite aerosols. J Quant Spectrosc Radiat Transfer 2011;112: 1815-24.

[22] Kahnert M, Rother T. Modeling optical properties of particles with small-scale surface roughness: combination of group theory with a perturbation approach. Opt Express 2011;19:1138-51.

[23] Kahnert M, Nousiainen T, Thomas MA, Tyynelä J. Light scattering by particles with small-scale surface roughness: comparison of four classes of model geometries. J Quant Spectrosc Radiat Transfer, http://dx.doi.org/10.1016/j.jqsrt.2012.03.017, this issue.

[24] Petrov D, Shkuratov Yu, Videen G. Light scattering by arbitrarily shaped particles having rough surfaces. J Quant Spectrosc Radiat Transfer, in this issue.

[25] Mackowski DW, Mishchenko MI. Calculation of the T matrix and the scattering matrix for ensembles of spheres. J Opt Soc Am A 1996;13:2266-78.

[26] Mackowski DW, Mishchenko MI. A multiple sphere T-matrix Fortran code for use on parallel computer clusters. J Quant Spectrosc Radiat Transfer 2011;112:2182-92.

[27] Mackowski DW 〈http://www.eng.auburn.edu/users/dmckwski/ scatcodes $>$.

[28] Mishchenko MI, Travis LD, Lacis AA. Scattering, absorption, and emission of light by small particles. Cambridge: Cambridge U. Press; 2002. Available at <http://www.giss.nasa.gov/staff/ mmishchenko/books.html $>$.

[29] Mishchenko MI, Travis LD, Kahn RA, West RA. Modeling phase functions for dustlike tropospheric aerosols using a shape mixture of randomly oriented polydisperse spheroids. J Geophys Res 1997;102:16831-47.

[30] Dubovik O, Sinyuk A, Lapyonok T, et al. Application of spheroid models to account for aerosol particle nonsphericity in remote sensing of desert dust. J Geophys Res 2006;111:D11208.

[31] Mishchenko MI, Travis LD. Light scattering by polydisperse, rotationally symmetric nonspherical particles: linear polarization. J Quant Spectrosc Radiat Transfer 1994;51:759-78.

[32] Volten H, Muñoz O, Rol E, et al. Scattering matrices of mineral aerosol particles at $441.6 \mathrm{~nm}$ and $632.8 \mathrm{~nm}$. J Geophys Res 2001;106:17375-402.

[33] Muñoz O, Moreno F, Guirado D, Ramos JL, Volten H, Hovenier JW. The IAA Cosmic Dust Laboratory: experimental scattering matrices of clay particles. Icarus 2011;211:894-900.

[34] Mishchenko MI, Hovenier JW. Depolarization of light backscattered by randomly oriented nonspherical particles. Opt Lett 1995;20: 1356-8. 